\title{
Electrode Interaction in Pediatric Cochlear Implant Subjects
}

\author{
Marc D. Eisen and Kevin H. Franck \\ Center for Childhood Communication, Children's Hospital of Philadelphia, Philadelphia, PA, USA \\ Department of Otorhinolaryngology, University of Pennsylvania Medical Center, Philadelphia, PA, USA
}

Received: 8 October 2004; Accepted: 16 February 2005; Online publication: 10 June 2005

\begin{abstract}
Multielectrode cochlear implants rely on differential stimulation of the cochlear nerve for presenting the brain with the spectral and timing information required to understand speech. In implant patients, the degree of overlap among cochlear nerve fibers stimulated by the different electrodes constitutes the electrode interaction. Electrode interaction degrades the spectral resolution of the implant's stimulus. We sought to define electrode interaction in a cohort of pediatric cochlear implant subjects as a function of both stimulus intensity and electrode location along the array. The 27 pediatric subjects that completed the study were implanted with either the Clarion HiFocus array with or without positioner, the Nucleus 24 Contour array, or the Nucleus 24 Straight array. All but two of the patients had congenital hearing loss, and none of the patients had meningitis prior to the onset of deafness. The cochlear nerve response was measured with the electrically evoked compound action potential (ECAP). A forward masking protocol was used such that a probe stimulus electrode remained fixed while a preceding masker was moved across the array. Electrode interaction was estimated by measuring the unmasked probe response minus the masked probe response. Three probe locations and three probe intensities were examined for each subject. At all probe locations, electrode interaction increased as probe intensity increased $(p<0.05)$. Interaction at the basal probe was less than that at either the middle or apical probe locations $(p<$
\end{abstract}

Correspondence to: Marc D. Eisen - Department of Otorhinolaryngology - University of Pennsylvania Medical Center 5 Ravdin/ORL, 3400 Spruce Street, Philadelphia, PA 19104, USA. Telephone: (215) 590-0531; fax: (215) 590-3986; email: eisenm@mail.med. upenn.edu
0.05 ), and significant correlation found between probe distance from the basal end of the array and electrode interaction $(p<0.001)$. These results demonstrate that in this cohort of pediatric subjects, electrode interaction depended on both stimulus intensity and probe location. Implications of these findings on future implant array design and current implant fitting strategies are discussed. The impact of electrode interaction on implant performance is yet to be elucidated.

Keywords: ECAP, electrode interaction, cochlear implant, pediatric

\section{INTRODUCTION}

Virtually all cochlear prostheses now employ a multiple electrode array. Sound coding schemes utilize multiple electrodes to break complex sounds into frequency components, with the goal of improving the spectral resolution of the signal that is presented to the auditory neural pathway. "Channel interaction" is the breakdown in discrimination of differences between electrodes at the level of perception. Two basic types of channel interactions are recognized-electrodes stimulated closely in time (temporal interaction) (Shannon 1983; White et al. 1984; Shannon 1990) and closely in space (spectral interaction) (Black and Clark 1980; White et al. 1984). Channel interaction is variable between subjects (White et al. 1984) and has been shown to have a significantly deleterious effect on speech recognition tasks (Throckmorton and Collins 2002; Boex et al. 2003). Conversely, decreasing channel interactions with sequential, rather than simultaneous, electrode stimulation has been a signal processing strategy that has resulted in substantial improve- 
ments in speech recognition (Wilson et al. 1991). Furthermore, improved channel discrimination has been associated with improved speech perception performance (Dorman et al. 1996; Collins et al. 1997; Dawson et al. 2000). At the level of perception, channel interaction has been primarily assessed through forward masking experiments, where elevated thresholds to stimuli preceded by a masker stimulus indicates interaction (White et al. 1984; Favre and Pelizzone 1993; de Balthasar et al. 2003). Interaction with this measure was found to be related to channel discrimination and pitch recognition (Cohen et al. 1996a). A subject's ability to discriminate the stimuli of different electrodes improves as the spatial separation of the electrodes increases, and the difference limen is slightly more than one electrode for most patients (Busby and Clark 1996; Collins et al. 1997).

A likely source of channel interaction is overlap in the populations of auditory nerve fibers stimulated by the electrodes. The degree of overlap in the subsets of stimulated auditory nerve fibers between electrodes is referred to as the interaction between those electrodes. Studies have addressed electrode interaction at the auditory nerve level. Direct measures of these types of electrode interaction have been measured in cat (Black and Clark 1980), rat (Haenggeli et al. 1998), and recently in a groups of adult human subjects with the Nucleus device (Cohen et al. 2003; Abbas et al. 2004). While these studies demonstrated the phenomenon of electrode interaction, small subject populations and varied durations and etiologies of deafness limited the power to evaluate trends in interaction along the implant's array. Most pediatric implant patients are prelingually deaf, have limited auditory experience, and are less likely to be willing to undergo behavioral testing. Objective testing is likely to benefit this patient cohort especially. We therefore sought to define electrode interaction in a cohort of pediatric cochlear implant subjects as a function of both stimulus intensity and electrode location along the array.

\section{MATERIALS AND METHODS}

Subjects

Pediatric implant recipients were recruited through a mailer and a follow-up phone call. No subjects dropped out of the study after having volunteered. Twenty-seven patients underwent the evoked compound action potential (ECAP) test battery. Twentyone of the 27 subjects were congenitally or perinatally deaf. Of the six other subjects, three had moderatesevere hearing loss that was initially aidable; these subjects had significant auditory experience prior to implantation. One subject's deafness was associated with a meduloblastoma that was treated with chemotherapy. Another subject's deafness had a sudden onset believed to have an autoimmune etiology. The etiology of the postlinguistically deafened subject was unknown. None of the subjects had meningitis prior to the onset of deafness, and all subjects had normal bony cochleae.

Their ages at implantation ranged from 0.7 to 12.9 (mean $=5.0 ; \mathrm{SD}=3.7$ years $)$. The subjects were tested between 2.9 and 41.4 months postimplantation (mean $=16.2 ; \mathrm{SD}=10.2$ months). Sixteen subjects were implanted with the Clarion CII implant (Advanced Bionics, Sylmar, CA) and 11 were implanted with the Nucleus 24 implant (Cochlear Limited, East Melbourne, Australia). Of the Clarion CII implants, nine included a silicone rubber positioner with the array, while seven had no positioner. Of the Nucleus 24 implant users, nine were implanted with the 24RCS Contour array, while two were implanted with the $24 \mathrm{M}$ straight array. Table 1 divides the subjects by electrode array type, summarizing patient age at implantation and time postimplantation at testing. This research was approved by the Institutional Review Board of the Children's Hospital of Philadelphia.

\section{ECAP measurements}

The reverse telemetry features of both the Nucleus and the Clarion implant were used to record ECAPs.

TABLE 1

\begin{tabular}{lcccc}
\hline \multicolumn{5}{c}{ TABLE 1 } \\
\hline Array type & Number & Mean age at implantation (SD) & Minimum age at implantation & Maximum age at implantation \\
\hline CN & 7 & $6.6(4.2)$ & 0.7 & 10.6 \\
CP & 9 & $4.0(3.0)$ & 1.2 & 10.8 \\
NC & 9 & $4.7(4.4)$ & 5.1 & 12.9 \\
NS & 2 & $5.3(0.1)$ & 0.7 & 5.3 \\
Totals & 27 & $5.0(3.7)$ & 12.9 \\
\hline
\end{tabular}

Minimum, maximum, and mean age at implantation is shown for subjects grouped by array type. Age is expressed in years.

$\mathrm{CP}=$ Clarion Hi-Focus array with positioner; $\mathrm{CN}=$ Clarion Hi-Focus array without positioner; $\mathrm{NC}=\mathrm{Nucleus}$ Contour array; $\mathrm{NS}=\mathrm{Nucleus}$ straight array. 
For the Clarion implant, reverse telemetry was performed with Neural Response Imaging (NRI) experimental software version V.42 (Advanced Bionics, Sylmar, CA); the Nucleus implant utilized Neural Response Telemetry (NRT) commercially released software version 3.0 (Cochlear Ltd., Lane Cove, Australia). The masker-probe algorithm (Brown et al. 1990) was utilized to subtract the stimulus artifact and isolate the ECAP. The masker and probe stimuli

A

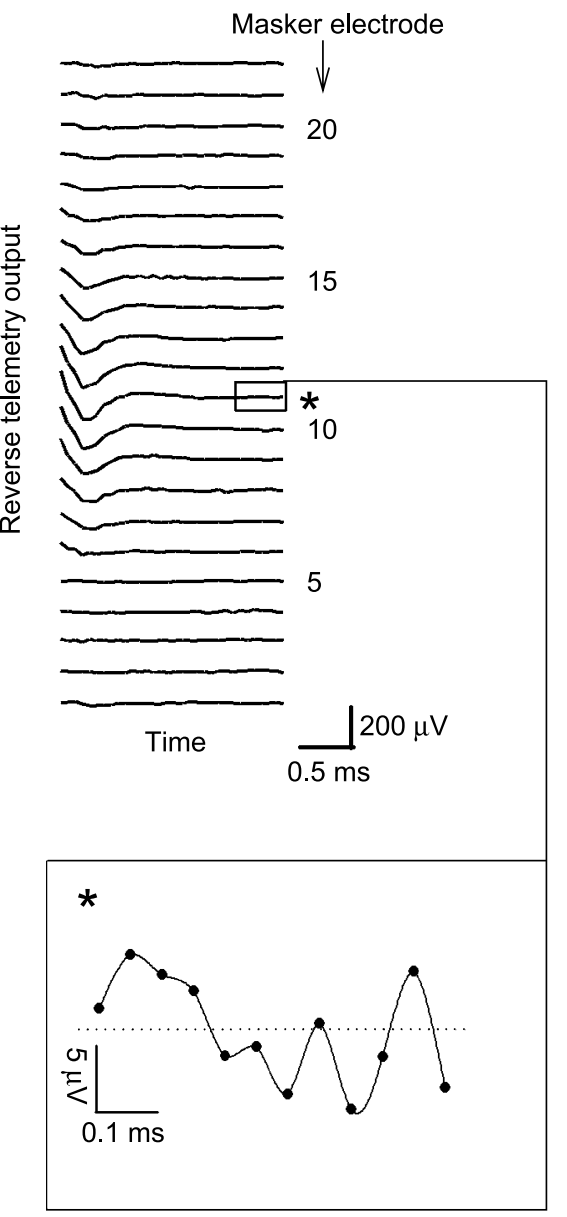

B

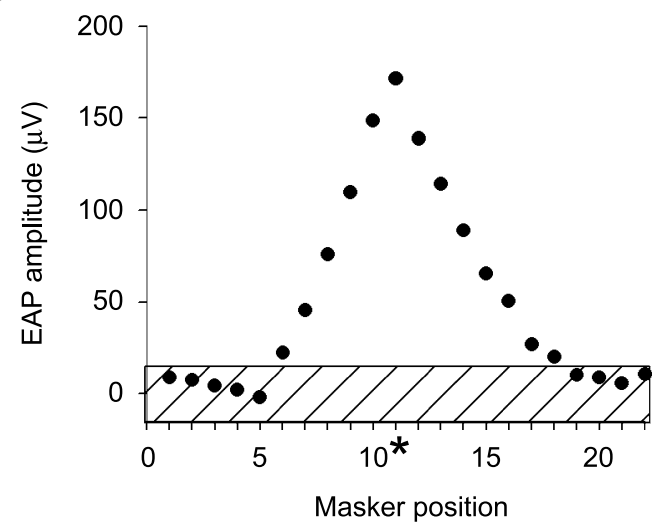

were biphasic negative leading pulses of equal phase duration. The masker preceded the probe by $500 \mu \mathrm{s}$ and had an intensity equal to the probe stimulus for the Nucleus subjects and $32 \mu \mathrm{A}$ higher than the probe stimulus for the Clarion subjects. Monopolar configuration was used for both stimulating and recording electrodes. Phase duration was $32 \mu$ s for the Clarion implant and $25 \mu$ s for the Nucleus implant. The recording electrode was located two electrodes in the apical direction from the probe. Gain was set to 300 for the Clarion CII and $60 \mathrm{~dB}$ for the Nucleus 24 implant. Each run was an average of at least 20 sweeps. ECAPs were digitized at $60 \mathrm{kHz}$ sampling rate with the CII's 9-bit analog-to-digital converter and at $20 \mathrm{kHz}$ with the Nucleus device. The initial depolarization peak, N1, and the subsequent hyperpolarization peak, $\mathrm{P} 1$, were chosen by visual inspection. ECAP magnitude was calculated as the difference between $\mathrm{P} 1$ and $\mathrm{N} 1$, and expressed in microvolts. Data were stored and analyzed off-line. Subjects were instructed to signal whether the stimuli were uncomfortably loud, and trials were abandoned if the subject demonstrated any signs of discomfort from the stimulus.

\section{Electrode interaction functions}

ECAP thresholds were first determined for the probe electrode by measuring an ECAP amplitude growth function, and extrapolating to zero amplitude (Franck 2002; Eisen and Franck 2004). ECAP threshold was then used as a reference magnitude to set the stimulus intensities for the interaction measurements in decibels relative to ECAP threshold. The electrode interaction waveform was measured by recording responses to a probe stimulus minus the response to a partially masked probe response. Artifact due to the masker is removed by subtracting a masker-only response. The greater the masker electrode extin-

FIG. 1. Example recording of interaction function in a subject implanted with the Nucleus 24RCS implant. The probe is electrode $\# 11$, and the recording electrode \#13. Both masker and probe have the same stimulus intensity. A Family of raw EAP traces for each masker location, where the masker electrode is labeled to the right of the trace. The trace where the probe and masker are on the same

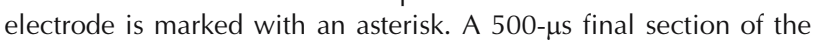
EAP recording from the condition where the masker and probe are both on electrode \#11 is enclosed with a box. This section is magnified below the traces, demonstrating the fluctuation of the ECAP recording. The dotted line represents the mean of the points during this time window. In this case, the standard deviation of the noise is $3.7 \mu \mathrm{V}$. This value multiplied by 4 was used to define the noise floor for the interaction function, as shown in B. B Example of an interaction function calculated from the traces in $\mathbf{A}$, where ECAP amplitude is shown as a function of masker electrode position. The noise floor, as calculated in $\mathbf{A}$, has been stippled. 
guishes the probe response, the greater the ECAP subtractive response and therefore the greater the interaction. Examples of these waveforms are shown in Figures 1 and 2. An "interaction function" resulted from the series of ECAP measurements made as above, but where the masker position was varied across the

A

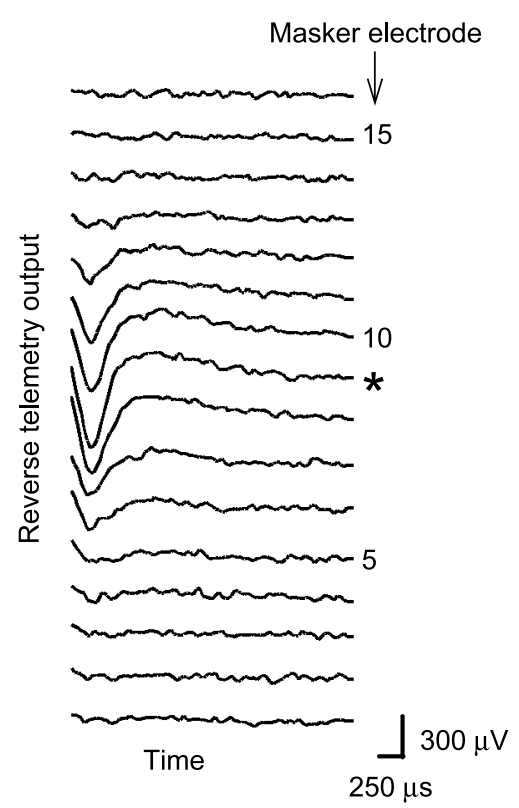

B
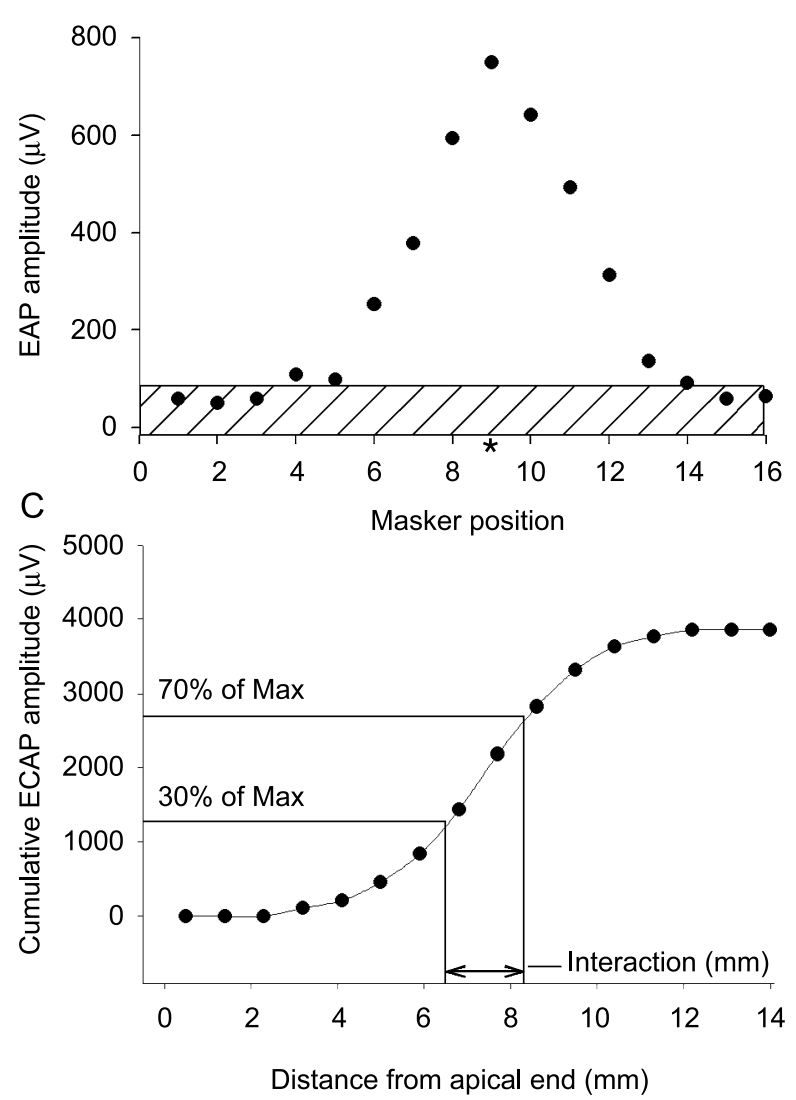

entire electrode array while the probe and recording electrode locations were fixed. Examples of interaction functions measured with both a Nucleus array (Fig. 1) and a Clarion array (Fig. 2) are demonstrated. All interaction functions had this general shape, where the magnitude of the ECAP subtractive response was greatest at or near the probe electrode.

\section{Interaction function analysis}

A noise floor for the interaction functions was calculated as four times the RMS noise level of the reverse telemetry output during a $300-\mu$ s unstimulated portion at the end of an epoch for the Clarion implant and a $500-\mu$ s portion for the Nucleus implant (Fig. 1) under the condition where the probe and masker were on the same electrode. This value was chosen so that the noise could be calculated for each specific probe electrode being tested. Four times the noise level was chosen because ECAPs with this amplitude were visually unequivocal. In order to quantify electrode interaction and compare between different implant manufacturers with different interelectrode distances, interaction functions were plotted as cumulative ECAP amplitude versus distance from the apical end of the array in millimeters (Fig. 2C). This cumulative function resembles an integration of interaction as a function of distance along the array.

ECAP magnitudes less than the calculated noise floor were counted as zero for the cumulative function. An example of a cumulative function derived from the interaction function in Figure 2B is demonstrated in Figure 2C. Interaction was then quantified as the distance along the electrode array (in millimeters) required to span the rise of the cumulative ECAP function from $30 \%$ to $70 \%$ of maximum cumulative ECAP value. This calculation is demonstrated in Figure 2C. Because the $30 \%$ and $70 \%$ points were invariably located between two electrodes, a linear interpolation was utilized between successive electrode points along the cumulative ECAP function. This value of the $30-70 \%$ distance will be referred

FIG. 2. Interaction function example from CII implant. ECAP raw traces $(\mathbf{A})$ and resulting interaction function $(\mathbf{B})$ from subject CP7 for the condition where the probe was at electrode \#9 and recording electrode at electrode \#7. Both probe and masker stimulus intensity are set at 528 current units, which is $2 \mathrm{~dB}$ above ECAP threshold. Noise floor was calculated as $81 \mu \mathrm{V}$ and shown as the stippled area in B. C Cumulative ECAP amplitude shown as a function of distance from the apical end of the electrode array. The masker position ( $x$ axis) in $\mathbf{B}$ corresponds to the distance from the apical end in $\mathbf{C}$. The points along the function at $30 \%$ and $70 \%$ of the maximum cumulative ECAP amplitude are marked, as well as the distance along the array spanned by these two points. We define this distance as the electrode interaction. 
A

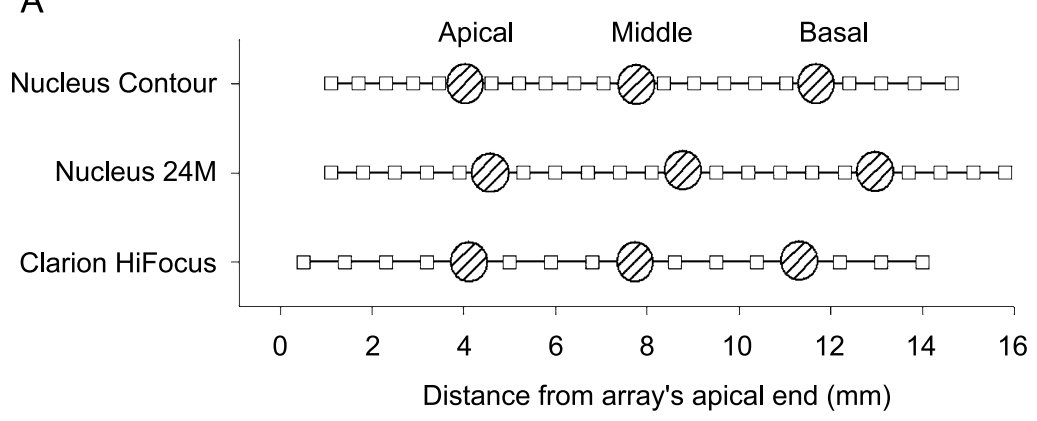

B

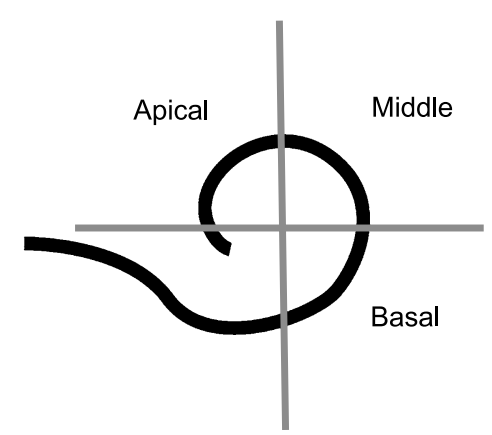

FIG. 3. Relative probe electrode location for three array configurations. A Location of all electrodes with respect to the apical end of the electrode array (squares) is shown for the three electrode arrays implanted in the subject population. Stippled circles demonstrate the three probe electrode locations used to measure interaction. B Schema of an inserted electrode array as seen via a "cochlear view" x-ray. The probe locations were chosen to represent each of the three quadrants of the first turn of the cochlea shown in this schema. The spiral represents the view of the electrode array in a cochlear view x-ray (Cohen et al. 1996a). The quadrants containing the apical, middle, and basal probe electrode locations are delineated by the gray lines and labeled accordingly. throughout this work as simply the "interaction" for a specific probe electrode.

A family of nine interaction functions was measured for each subject: Functions were obtained at three stimulus intensities closest to 2, 3, and $4 \mathrm{~dB}$ above ECAP threshold, and at three different probe locations along the electrode array. Lower intensities were measured prior to higher intensities. Intensity

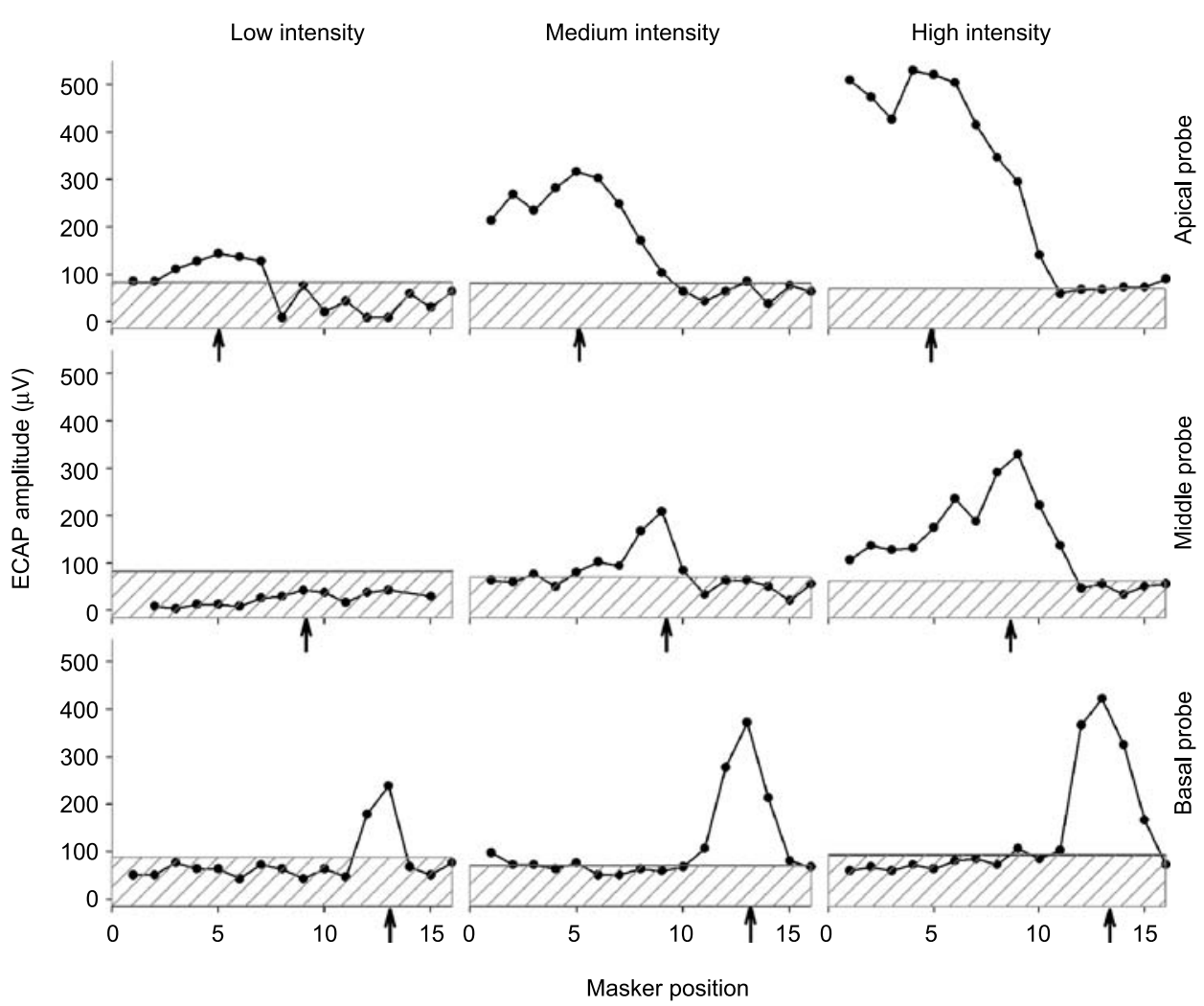

FIG. 4. Family of ECAP interaction functions from one subject. Interaction functions at all three stimulus intensities and three probe locations from subject CP3 are shown, where ECAP amplitude is shown as a function of masker electrode position (apicalmost electrode = \#1). Each of the nine panels is a single interaction function at a single intensity and probe location. Panels are arranged such that relative stimulus intensity is arranged in columns, and probe location arranged by rows. Arrows point to the probe location. Note the typical asymmetric interaction function for apical probe electrodes. 

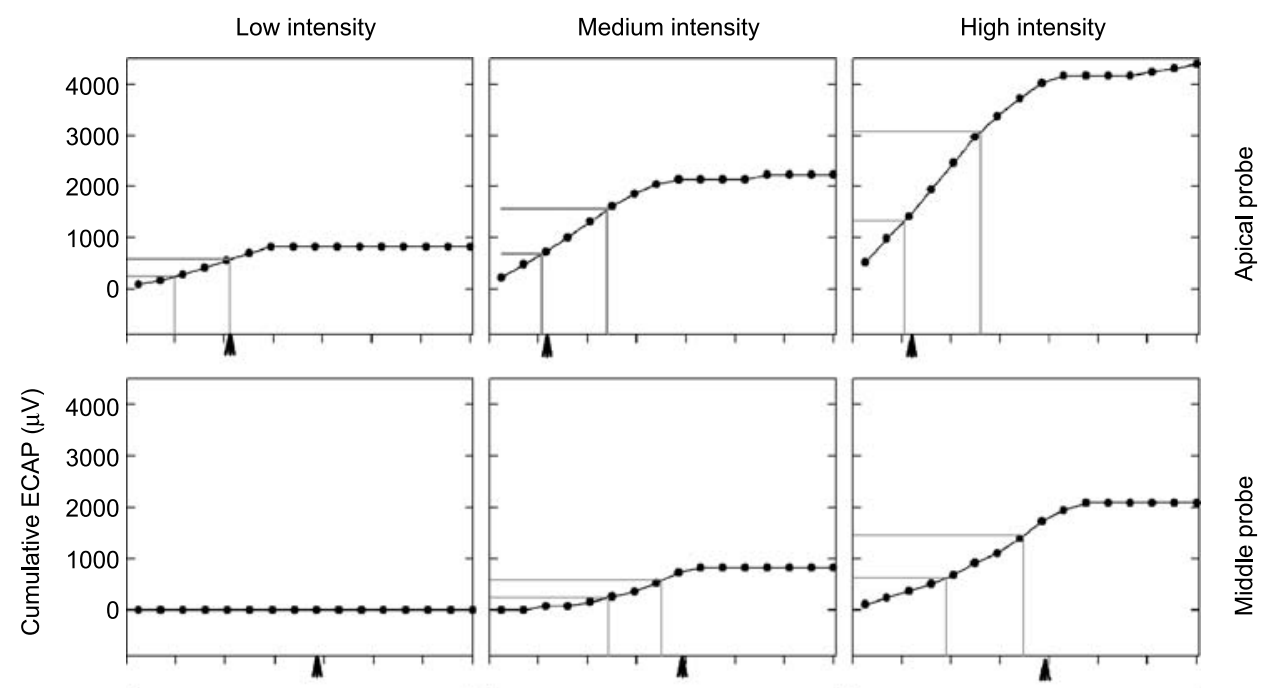

\section{$\frac{0}{0}$
$\frac{0}{0}$
$\frac{0}{0}$
$\frac{0}{\overline{0}}$
$\frac{0}{2}$}

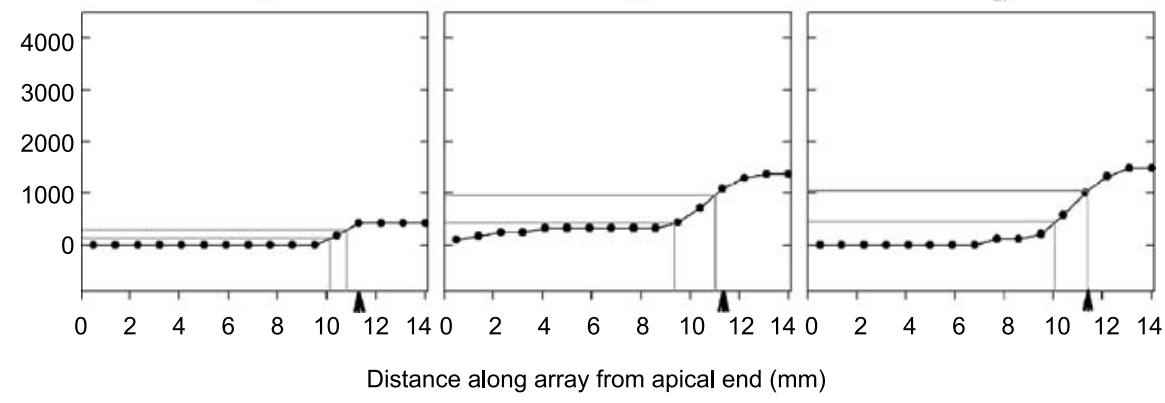

FIG. 5. Analysis of one subject's electrode interaction. Cumulative ECAP amplitude is first calculated as a function of distance (in millimeters) along the electrode array, as shown for the corresponding panels from the interaction functions in Figure 4. The points along the function whose cumulative ECAP value is $30 \%$ and $70 \%$ of the maximum value are shown as gray lines. The corresponding interaction is defined by the distance along the array between the two vertical gray lines. Arrowheads mark the position of the masker electrode for reference.

was measured as $\mathrm{dB}$ relative to ECAP threshold because the ECAP threshold is an objective measurement of auditory nerve response to electrical stimulation independent of behavioral measures. It should be noted that the stimulus amplitude could rarely be raised high enough in these subjects to reach a plateau for the amplitude growth functions, due to the discomfort of loud stimuli. Dynamic range is therefore typically not measurable.

We tested three probe electrode positions for each array type. These probe electrode positions are displayed as distance along the electrode array relative to the array's apical end in Figure 3A for each array type. These positions were chosen so as to test one electrode from each of the quadrants demonstrated in Figure $3 \mathrm{~B}$, defined by the electrode insertion angle described in several publications (Cohen et al. 1996b; $\mathrm{Xu}$ et al. 2000; Balkany et al. 2002). The center-tocenter interelectrode spacing of the Clarion CII implant is $0.9 \mathrm{~mm}$, and that of the Nucleus $24 \mathrm{M}$ straight array is $0.7 \mathrm{~mm}$. For the Nucleus Contour array, the interelectrode spacing is not uniform and ranges between 0.57 and $0.81 \mathrm{~mm}$. The location of the electrodes along each of these three array types with respect to the apical end of the array, as well as the specific probe electrodes utilized, is shown in Figure 3A. For the Clarion subjects, the three probe locations were electrodes \#5 (apical), \#9 (middle), and \#13 (basal). For the Nucleus subjects, probe electrodes \#17 (apical), \#11 (middle), and \#5 (basal) were used. Nine of the 27 patients had postoperative $\mathrm{x}$-rays available for review. We used these studies as representative samples of the electrode locations of the different array types. The arrays represented by these nine patients included the Clarion Hi-Focus array with and without positioner and the Nucleus Contour array. All radiographs confirmed the reported "full" insertions. Using the method of defining the angle of insertion of electrodes described by Cohen et al. (1996b), all probe locations fell within the quadrants specified in Figure 3B. This is corroborated by Balkany et al. (2002), who examined the electrode insertion angle of the Clarion Hi-Focus array with positioner and Nucleus Contour array with videofluoroscopy. An example of the nine interaction functions measured for one representative subject is shown in Figure 4, with the corresponding cumulative functions displayed in Figure 5.

\section{RESULTS}

\section{Electrode interaction}

Electrode interaction, calculated in millimeters as demonstrated in Figure 2C, was measured for all subjects at three probe intensities and three probe 


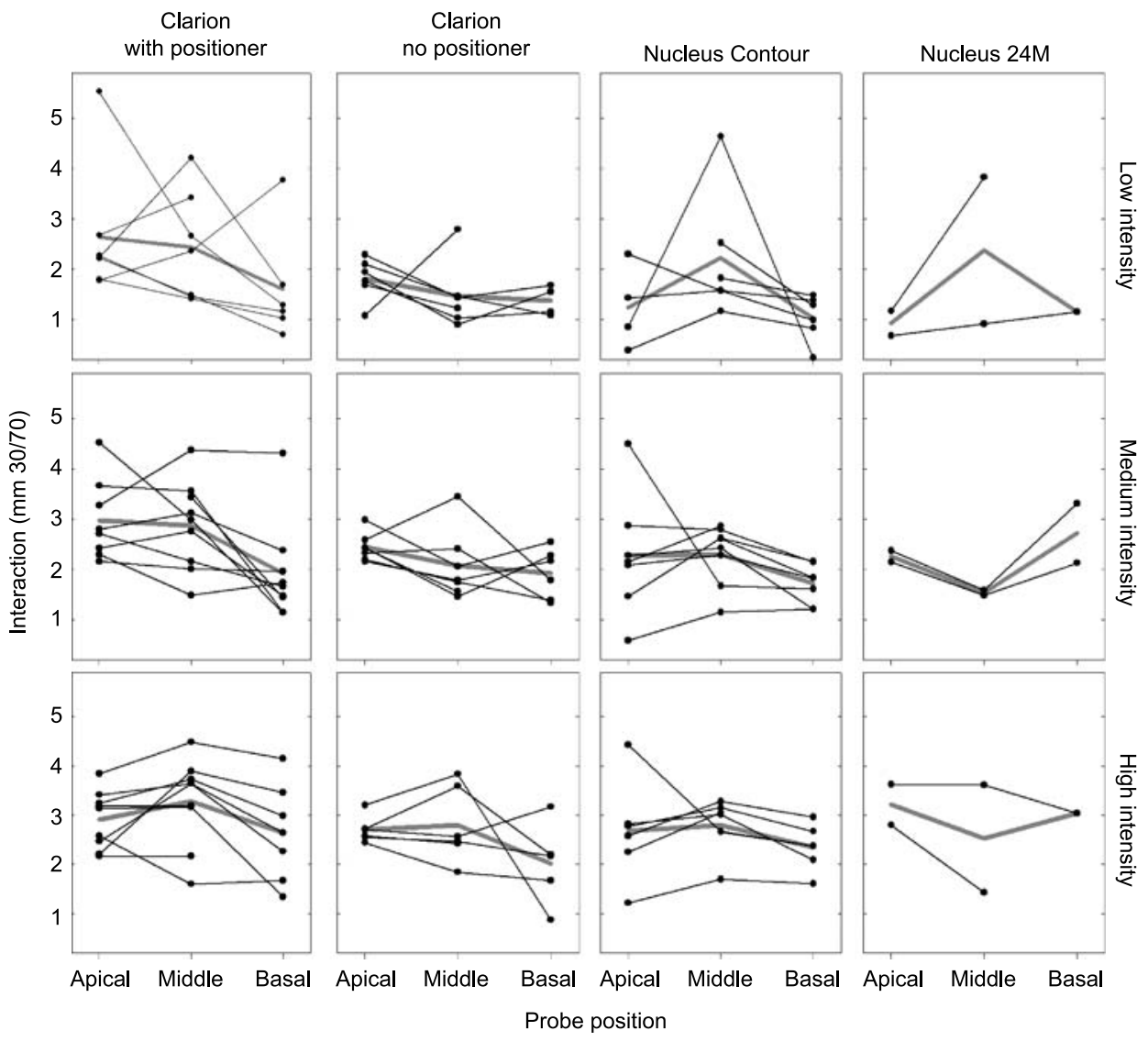

FIG. 6. Trends of electrode interaction in individual subjects at different probe locations. Each panel represents a group of subjects with the same array configuration and stimulus intensity. Subjects with the same electrode configuration are arranged in columns, while stimulus intensity is arranged in rows. Lines connect values of electrode interaction for the three different probe locations - apical, middle, and basal. Missing points occur where interaction could not be measured. Mean values are shown by the gray line. locations similar to that shown in Figure 4. The interaction values for the three probe locations of each subject are demonstrated in Figure 6, parsed by intensity and array type. Values of interaction reveal considerable variability between subjects.

\section{Effect of intensity on interaction}

Examining the interaction values in Figure 6 within columns of increasing probe intensity may suggest increasing interaction with increasing intensity. In order to quantify the effect of stimulus intensity on interaction, interaction values for all subjects were grouped by stimulus intensity (as low, medium, and high) and probe location (as apical, middle, and basal), and the mean values ( \pm SEM) for each category displayed in Figure 7. At each probe location, mean interaction increased as stimulus intensity increased. Bivariate association among the three stimulus intensities was assessed using Spearman's rank correlation (exact version). Correlation coefficients relating stimulus intensity to interaction were $0.563,0.359$, and 0.606 for the apical, middle, and basal probe, respectively, and all were significant $(p<0.05)$. Pairwise comparison of interaction between individual intensities at each probe location reveals significant differences $(p<0.05)$ between low- and high-intensity stimuli at all three probe locations (Tukey test). Although the mean interaction at medium stimulus intensity fell between the value at low and high in-

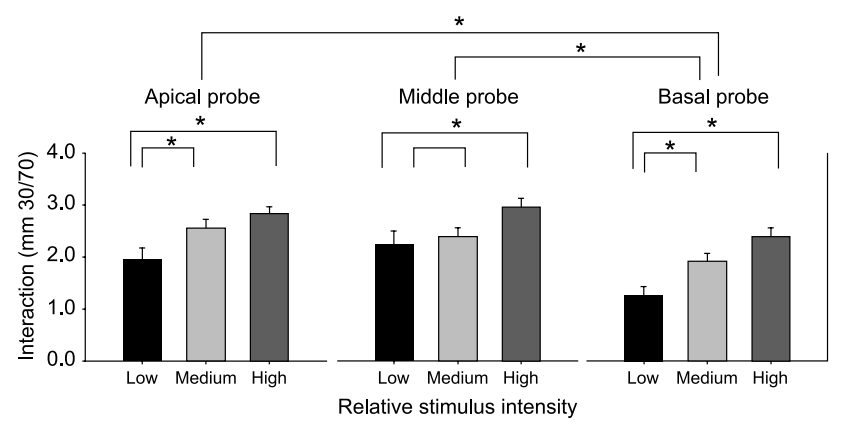

FIG. 7. Summary of electrode interaction at different probe locations and intensities. Vertical bars represent mean values of interaction for all subjects at three different intensities and three different probe locations. Error bars denote standard error. Differences in interaction between intensities for each probe location are marked with an asterisk $(*)$ if the $p$ value of the difference ( $t$ test) is less than 0.05. Comparison between probe electrode locations collapsed across intensities is shown at the top of the figure. $N=81$, 81 , and 80 for the apical, middle, and basal probe locations. Asterisks denote significant differences between groups (Tukey test, $p<0.05$ ). 
tensity, significant differences were found only between low and medium intensity at the apical and basal probe locations (Fig. 7). Stimulus intensity therefore appears to be an important determinant of electrode interaction.

\section{Effect of probe location}

Figure 4 shows wider interaction functions (and hence more electrode interaction) at apical probe locations than at basal probe locations. Furthermore, a trend of decreasing interaction within subjects can be observed in the individual results of Figure 6 . We therefore addressed the contribution of probe location to electrode interaction (Fig. 7). Despite considerable individual variation, interaction at the basal probe location tended to be lower than that at the apical location. Statistically significant negative correlation was found between interaction and distance of the probe from the apical end of the array, independent of differences due to intensity $(\rho=$ $-0.280 ; p<0.001$, Spearman test). Paired comparison of interaction for all subjects also revealed that interaction at the basal end of the array [1.87 \pm 0.11 (SEM) $\mathrm{mm}, n=80$ ] is less than that at the apical and middle locations, respectively $(2.46 \pm 0.11 \mathrm{~mm}, n=81$ and $2.53 \pm 0.11 \mathrm{~mm}, n=81, p<0.05$, Tukey test) (Fig. 7). This relationship between probe location and interaction may be seen in the mean values of interaction at each probe location for all subjects and intensities (Fig. 7). Interaction therefore appears to be greater at the apical end of the array compared to the basal end.

\section{Effect of array configuration on electrode interaction}

Four different electrode array configurations are represented by the subjects in the study, as shown in Table 1: the Clarion Hi-Focus I array with positioner, the Clarion Hi-Focus I array without positioner, the Nucleus 24RCS Contour array, and the Nucleus 24M straight array. A comparison of electrode interaction among the Clarion Hi-Focus I array with and without positioner and the Nucleus Contour array is shown for the three different probe locations in Figure 8. The two subjects with the Nucleus 24M straight array were not included in the comparison because of the small sample size. Two-way analysis of variance (ANOVA) of interaction with respect to array type and location reveals significant effects of array type on electrode interaction $(p<0.05)$ and no interaction between array type and probe location. Differences in interaction among array types were greater at the apical and middle probe locations than at the

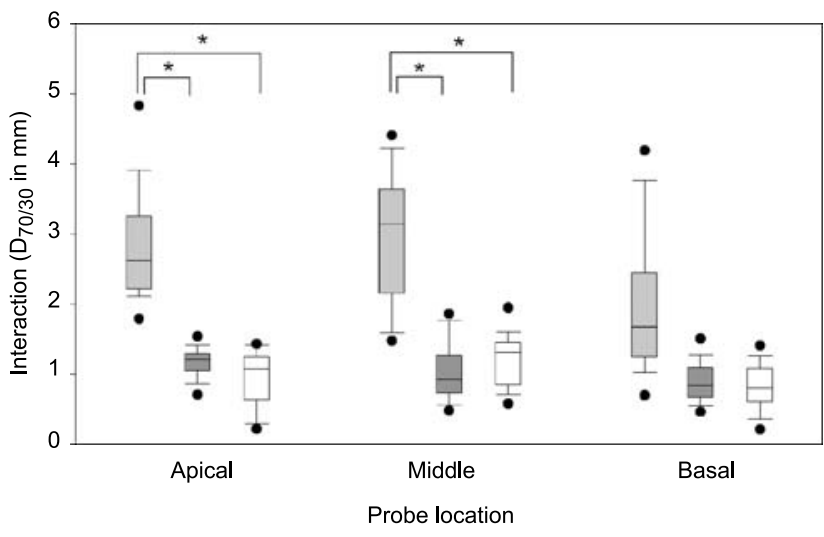

FIG. 8. Influence of array configuration on electrode interaction. Box plot showing interaction for subjects with the Clarion Hi-Focus I array with positioner (light gray), Hi-Focus I array without positioner (dark gray), or Nucleus Contour array (white) at the three different probe locations. Asterisk depicts significant differences between groups $(p<0.05)$. Box plots represent the median value, the 25 th and 75th percentile (box), the 10th and 90th percentile (error bars), and minimum and maximum values (dots).

basal probe location. At both the apical and middle probe locations, subjects with the Clarion Hi-Focus I array with the positioner demonstrated more interaction than the Hi-Focus I array without the positioner or the Nucleus Contour array $(t$ test, $p<0.05$ ). No differences in interaction were evident between the Clarion Hi-Focus I array without positioner and the Nucleus Contour array.

\section{DISCUSSION}

The electrodes along a multielectrode array are designed to stimulate a discrete population of the surviving auditory nerve fibers. The degree of overlap of these populations among the different electrodes along the array determines the degree of the implant's electrode interaction. Electrode interaction can result from both a summation of current spread among electrodes and by the overlap of neural populations excited by different electrodes. While current summation as a source of electrode interaction has improved with the implementation of nonsimultaneous stimulation strategies (Wilson et al. 1988; Favre and Pelizzone 1993), few studies have directly evaluated the contribution of overlapping excited neural populations to channel interaction. The contribution of overlapping neural populations to electrode interaction was directly measured in this work by using the ECAP measurement with the masker-probe paradigm. With this technique we showed in a cohort of pediatric subjects that elec- 
trode interaction generally increases with increasing intensity and is greater toward the apical end compared to the basal end of the electrode array. These findings have both basic science and clinical implications.

As the exact angular location of the electrodes within the cochlea has some variability between subjects as measured by videofluoroscopy (Balkany et al. 2002), we chose not to specifically localize each probe electrode location for each subject but rather to broadly choose electrodes that would reliably be found in the quadrants depicted in Figure 3B. The disadvantage of this approach is that the longitudinal location of electrodes cannot be ruled out as a contributor to the differences in interaction seen between subjects and array types. However, the location of the electrodes along the arrays is documented and accounted for in the analysis of interaction.

\section{Quantifying interaction}

The interaction function is composed of single data points for each electrode along the array, which yields at most 16 or 22 points depending on the array. We sought an analysis of the interaction function that could accommodate the function's limited resolution and at the same time allow a direct comparison among the electrode arrays with disparate interelectrode separations. The analysis that met these criteria was the $30-70 \%$ distance of the cumulative ECAP interaction function. This measurement proved to be a robust measurement of interaction and that could be directly compared between different array designs. An alternative analysis of the interaction function that has been described is to measure the function's width at a specified relative height (Cohen et al. 2003; Abbas et al. 2004). We found that with the Clarion array especially (16 points), this type of analysis suffered from the limited number of points such that small changes in individual data points significantly altered the measurement. Nevertheless, when this analysis was used, electrode interaction from the base to the apex was similarly found to increase $(p<0.001$, Spearman's exact test, data not shown).

\section{Gradient in interaction along length of cochlea}

One of the significant findings in this work was that electrode interaction was greater for apical than for basal electrodes. This finding suggests a gradient in the source of electrode interaction along the length of the cochlea in congenital deafness. An interaction gradient was not observed in similar studies of electrode interaction in adult subjects (Cohen et al. 2003;
Abbas et al. 2004). Possible sources of gradients along the cochlea's length include the density and excitability of surviving ganglion cells, the anatomic relationship between the electrodes and the ganglion cells, and the impedance along the length of the cochlea. A histologic study of temporal bones from normal hearing individuals shows a lower ganglion cell density at the cochlear apex compared to the base (Ariyasu et al. 1989). Deafness has been associated with a greater loss of cochlear ganglion cells toward the basal aspect of the cochlea compared to the apex (Nadol 1997). However, the subjects in this study had variable onset and etiology of their deafness, which may yield a different pattern of ganglion cell loss compared to a more uniform group of pediatric subjects primarily with congenital deafness. It is unclear whether a greater density of excitable ganglion cells would in itself lead to greater or lesser electrode interaction. Correlating electrode interaction with histological evaluation will help elucidate this relationship.

Gradients in the cross-sectional anatomy of the cochlea along its length demonstrate that the distance between the medial wall of scala tympani and the ganglion cells increases from the base to the apex of the cochlea (Ariyasu et al. 1989). A model of these gradients predicted that thresholds should decrease while cross-turn stimulation and growth function slopes increase from the base toward the apex (Frijns et al. 2001). This prediction was supported by data from pediatric subjects implanted with the Clarion CII implant (Eisen and Franck 2004). An increase in cross-turn stimulation toward the cochlear apex would be expected to increase the degree of electrode interaction from the base to apex; this is consistent with our findings. However, cross-turn stimulation would be expected to increase substantially with increases in intensity, which we did not see. Differences in the spatial attenuation of current within the cochlea (Kral et al. 1998) along its length may also contribute to the gradient of electrode interaction. Finally, systematic differences in the excitability of ganglion cells along the cochlea's length has precedence in murine cochlear ganglion cells (Adamson et al. 2002) and could also contribute to interaction gradients.

\section{Relationship of electrode interaction to channel independence}

The psychophysical consequences of electrode interaction have been addressed in several studies. As we have shown here for electrode interaction, channel interaction has generally been found to decrease with increasing separation of the masker and the probe 
electrodes. In a group of adult implant listeners, for example, forward masking at different electrodes along the implant's array was most effective at increasing psychophysical thresholds when the masker and probe were on the same electrode, and the degree of forward masking correlated with pitch perception differences between subjects (Boex et al. 2003). In a small study of five implanted adults, subjects could discriminate pitch differences between electrodes more consistently as the interelectrode separation increased (Carlyon et al. 2000).

\section{Role of perimodiolar electrode array positioning}

The silicone rubber positioner and precurved array force the electrodes closer to the modiolus (Fayad et al. 2000) and thus theoretically closer to the excitable neural elements. Closer electrodes should not only reduce the amount of electric current necessary for stimulation (Franck and Norton 2001), but may also decrease the interaction between electrodes. One study looked at the perturbation of probe thresholds with different maskers and found no difference in interaction between those subjects with and those without the silicone rubber positioner in monopolar configuration (Boex et al. 2003). In a previous study, we reported that ECAP thresholds were lower with than without the positioner, a finding that supported a closer proximity between the electrodes and excitable neural elements with the positioner. Additionally, no trend in ECAP threshold across the length of the electrode array was evident (Eisen and Franck 2004). We report here, in the same subject population, no significant decrease in electrode interaction with the silicone rubber positioner. To the contrary, interaction was greater in the subjects with the positioner at the apical and middle probe locations, on average. These results do not support the hypothesis that the positioner decreases electrode interaction. Explanations would include the possibility that having the excitable neural elements closer to the stimulating array would lead to increases, rather than decreases, in electrode interaction. Additionally, the positioner moves the electrodes not only closer to the modiolus, but also deeper into the cochlea. Because electrode interaction appears to be greater toward the apical end of the electrode array, as we have shown here, then systematically deeper insertions with the positioner may yield greater interaction. This hypothesis would be addressed by a more detailed localization of the electrodes with imaging. Further studies that correlate electrode interaction with channel discrimination at the level of perception may help elucidate the effectiveness of the perimodiolar electrode positioning.

\section{Clinical implications}

A wide subject-to-subject variability exists in speech recognition abilities of children with cochlear implants, despite similar speech processing schemes and implant hardware. Differences in electrode interaction between subjects may explain this variability (Friesen et al. 2001). Minimizing electrode interaction is likely to enhance channel discrimination and improve implant performance on more challenging acoustic tasks such as listening to speech in noisy environments or appreciating music; future studies are needed to elucidate the clinical importance of electrode interaction. If electrode interaction is shown to contribute to implant performance, our demonstration here that electrode interaction toward the cochlear apex is greater than that of the cochlear base may have implications for implant design in the pediatric population. Electrode interaction may be minimized by increasing the density of electrodes at the basal end of the array, or speech processing schemes can be manipulated to preferentially utilize the basal electrodes over the apical electrodes.

\section{Monopolar versus bipolar stimulation}

These studies were done using the monopolar, as opposed to a bipolar, electrode configuration. The monopolar configuration utilizes a distant ground electrode, while the bipolar configuration utilizes an adjacent electrode as the ground. Monopolar configuration yields widespread current density while bipolar yields narrower current density. Bipolar stimulation should therefore result in less electrode interaction. Changing from a monopolar to bipolar configuration has not been demonstrated to affect speech recognition, and subjects tended to prefer the monopolar configuration when presented with both (Miller et al. 2001). Therefore, while bipolar stimulation has been shown to reduce electrode interaction (Bierer and Middlebrooks 2004; Pfingst and Xu 2004), it may not improve the sound quality that the implant subject perceives. Electrode interaction and its relationship to other stimulation parameters that affect sound perception will help elucidate what may appear to be contradictions.

\section{CONCLUSIONS}

In a cohort of pediatric cochlear implant subjects, electrode interaction was directly measured using the ECAP and found to depend on stimulus intensity and probe location. Increasing intensity and apical probe 
locations were associated with increasing electrode interaction. This finding has both basic and clinical implications. The contribution of electrode interaction to channel independence and implant performance has yet to be elucidated.

\section{ACKNOWLEDGMENTS}

This work was graciously supported by the ENT division of the Children's Surgical Associates and a Resident Research Grant from the Academy of Otolaryngology (M.D.E).

\section{REFERENCES}

Abbas PJ, Hughes Ml, Brown CJ, Miller CA, South H. Channel interaction in cochlear implant users evaluated using the electrically evoked compound action potential. Audiol. Neurootol. 9:203-213, 2004.

Adamson CL, Reid MA, Davis RL. Opposite actions of brain-derived neurotrophic factor and neurotrophin-3 on firing features and ion channel composition of murine spiral ganglion neurons. J. Neurosci. 22:1385-1396, 2002.

Ariyasu L, Galey FR, Hilsinger RJ, Byl FM. Computer-generated three-dimensional reconstruction of the cochlea. Otolaryngol. Head Neck Surg. 100:87-91, 1989.

Balkany TJ, Eshraghi AA, Yang N. Modiolar proximity of three perimodiolar cochlear implant electrodes. Acta Otolaryngol. 122:363-369, 2002.

Bierer JA, Middlebrooks JC. Cortical responses to cochlear implant stimulation: channel interactions. Jaro 5:32-48, 2004.

Black RC, Clark GM. Differential electrical excitation of the auditory nerve. J. Acoust. Soc. Am. 67:868-874, 1980.

Boex C, Kos M, Pelizzone M. Forward masking in different cochlear implant systems. J. Acoust. Soc. Am. 114:2058-2065, 2003.

BRown CJ, ABBAS PJ, GANTZ B. Electrically evoked whole-nerve action potentials: data from human cochlear implant users. J. Acoust. Soc. Am. 88:1385-1391, 1990.

Busby PA, Clark GM. Electrode discrimination by early-deafened cochlear implant patients. Audiol. 35:8-22, 1996.

Carlyon RP, Geurts L, Wouters J. Detection of small acrosschannel timing differences by cochlear implantees. Hear. Res. 141:140-154, 2000.

Cohen LT, Busby PA, Clark GM. Cochlear implant place psychophysics. 2. Comparison of forward masking and pitch estimation data. Audiol. Neuro-otol. 1:278-292, 1996a.

Cohen LT, Xu J, Xu SA, Clark GM. Improved and simplified methods for specifying positions of the electrode bands of a cochlear implant array. Am. J. Otol. 17:859-865, 1996b.

Cohen LT, Richardson LM, Saunders E, Cowan RS. Spatial spread of neural excitation in cochlear implant recipients: Comparison of improved ECAP method and psychophysical forward masking. Hear. Res. 179:72-87, 2003.

Collins LM, Zwolan TA, Wakefield GH. Comparison of electrode discrimination, pitch ranking, and pitch scaling data in postlingually deafened adult cochlear implant subjects. J. Acoust. Soc. Am. 101:440-455, 1997.

Dawson PW, McKay CM, Busby PA, Grayden DB, Clark GM. Electrode discrimination and speech perception in young children using cochlear implants. Ear Hear. 21:597-607, 2000.

De Balthasar C, Boex C, Cosendai G, Valentini G, Sigrist A, Pelizzone M. Channel interactions with high-rate biphasic electrical stimulation in cochlear implant subjects. Hear. Res. 182:77-87, 2003.

Dorman MF, Smith LM, Smith M, Parkin JL. Frequency discrimination and speech recognition by patients who use the Ineraid and continuous interleaved sampling cochlear-implant signal processors. J. Acoust. Soc. Am. 99:1174-1184, 1996.

EISEN MD, FRANCK KH. ECAP amplitude growth functions and high resolution programming levels in pediatric CII implant subjects. Ear Hear. 25:528-538, 2004.

Favre E, Pelizzone M. Channel interactions in patients using the Ineraid multichannel cochlear implant. Hear. Res. 66:150-156, 1993.

FAyAd JN, Luxford W, Linthicum FH. The Clarion electrode positioner: temporal bone studies. Am. J. Otol. 21:226-229, 2000.

FrancK KH. A model of a nucleus 24 cochlear implant fitting protocol based on the electrically evoked whole nerve action potential. Ear Hear. 23:67S-71S, 2002.

Franck KH, Norton SJ. Estimation of psychophysical levels using the electrically evoked compound action potential measured with neural response telemetry capabilities of Cochlear Corporation's CI24M Device. Ear Hear. 22:289-299, 2001.

Friesen LM, Shannon RV, Baskent D, WANG X. Speech recognition in noise as a function of the number of spectral channels: comparison of acoustic hearing and cochlear implants. J. Acoust. Soc. Am. 110:1150-1163, 2001.

FrIJNS JHM, Briaire JJ, Grote JJ. The importance of human cochlear anatomy for the results with modiolus hugging multi-channel cochlear implants. Otol. Neurotol. 22:340-349, 2001.

Haenggeli A, Zhang JS, Vischer MW, Pelizzone M, Rouiller EM. Electrically evoked compound action potential (ECAP) of the cochlear nerve in response to pulsatile electrical stimulation of the cochlea in the rat: effects of stimulation at high rates. Audiol. 37:353-371, 1998.

Kral A, Hartmann R, Mortazavi D, Klinke R. Spatial resolution of cochlear implants: the electrical field and excitation of auditory afferents. Hear. Res. 121:11-28, 1998.

Miller Al, Arenberg JG, Middlebrooks JC, Pfingst BE. Cochlear implant thresholds: comparison of middle latency responses with psychophysical and cortical-spike-activity thresholds. Hear. Res. 152:55-66, 2001.

NADOL JB JR. Patterns of neural degeneration in the human cochlea and auditory nerve: implications for cochlear implantation. Otolaryngol. Head Neck Surg. 117:220-228, 1997.

Pfingst BE, Xu L. Across-site variation in detection thresholds and maximum comfortable loudness levels for cochlear implants. Jaro 5:11-24, 2004.

SHANNON RV. Multichannel electrical stimulation of the auditory nerve in man. II. Channel interaction. Hear. Res. 12:1-16, 1983.

SHANNON RV. Forward masking in patients with cochlear implants. J. Acoust. Soc. Am. 88:741-744, 1990.

Throckmorton CS, Collins LM. The effect of channel interactions on speech recognition in cochlear implant subjects: predictions from an acoustic model. J. Acoust. Soc. Am. 112:285-296, 2002.

White MW, Merzenich MM, Gardi JN. Multichannel cochlear implants. Channel interactions and processor design. Arch. Otolaryngol. 110:493-501, 1984.

Wilson BS, Finley CC, Farmer JC Jr, Lawson DT, Weber BA, Wolford RD, Kenan PD, White MW, Merzenich MM, Schindler RA. Comparative studies of speech processing strategies for cochlear implants. Laryngoscope 98:1069-1077, 1988.

Wilson BS, Finley CC, Lawson DT, Wolford RD, Eddington DK, Rabinowitz WM. Better speech recognition with cochlear implants. Nature 352:236-238, 1991.

Xu J, Xu SA, Cohen LT, Clark GM. Cochlear view: postoperative radiography for cochlear implantation. Am. J. Otol. 21:49-56, 2000 . 